\title{
Hepatic Lipid Accumulation Induced by a High-fat Diet Is Regulated by Nrf2 Through Multiple Pathways
}

\section{sheng Qiu}

Chongqing Medical University

\section{Zerong Liang}

Chongqing Medical University

\section{Qinan Wu}

Chongqing Medical University

\section{Miao Wang}

Chongqing Medical University

\section{Mengliu Yang}

Chongqing Medical University

\section{Chen Chen}

UQ SBMS: The University of Queensland School of Biomedical Sciences

\section{Hong ting zheng}

Third Military Medical University Second Affiliated Hospital: Xinqiao Hospital

\section{Zhiming Zhu}

Third Military Medical University Daping Hospital

\section{Ling Li}

Chongqing University of Medical Science: Chongqing Medical University

\section{Gangyi Yang ( $\sim$ gangyiyang@163.com )}

Chongqing Medical University

\section{Research}

Keywords: Nrf2, lipid accumulation, MAFLD, SREBP-1, autophagy

Posted Date: August 17th, 2021

DOI: https://doi.org/10.21203/rs.3.rs-775710/v1

License: (1) (1) This work is licensed under a Creative Commons Attribution 4.0 International License.

Read Full License 
Version of Record: A version of this preprint was published at The FASEB Journal on April 8th, 2022. See the published version at https://doi.org/10.1096/fj.202101456R. 


\section{Abstract}

\section{Background}

Nuclear factor erythroid 2-related factor 2 (Nrf2) is reportedly involved in hepatic lipid metabolism, but the results are contradictory and the underlying mechanism thus remains unclear. Herein we focused on elucidating the effects of Nrf2 on hepatic adipogenesis and on determining the possible underlying mechanism. We established a metabolic associated fatty liver disease (MAFLD) model in high fat diet (HFD) fed Nrf2 knockout (Nrf2 KO) mice; further, a cell model of lipid accumulation was established using mouse primary hepatocytes (MPHs) treated with free fatty acids (FAs). Using these models, we investigated the relationship between Nrf2 and autophagy and its role in the development of MAFLD.

Results

We observed that Nrf2 expression levels were up-regulated in patients with MAFLD and diet-induced obese mice. Nrf2 deficiency led to hepatic lipid accumulation in vivo and in vitro, in addition to, promoting lipogenesis mainly by increasing SREBP-1 activity. Moreover, Nrf2 deficiency attenuated autophagic flux and inhibited the fusion of autophagosomes and lysosomes in vivo and in vitro. Weakened autophagy caused reduced lipolysis in the liver. Importantly, Chromatin immunoprecipitation-qPCR (ChIP-qPCR) and dual-luciferase assay results proved that Nrf2 bound to LAMP1 promoter and regulated its transcriptional activity. We accordingly report that Nrf2-LAMP1 interaction has an indispensable role in Nrf2-regulated hepatosteatosis.

\section{Conclusions}

These data collectively confirm that Nrf2 deficiency promotes hepatosteatosis by enhancing SREBP-1 activity and attenuating autophagy. To conclude, our data reveal a novel multi-pathway effect of Nrf2 on lipid metabolism in the liver, and we believe that multi-target intervention of Nrf2 signaling is a promising new strategy for the prevention and treatment of MAFLD.

\section{Introduction}

Metabolic associated fatty liver disease (MAFLD) has become a major health problem in humans and an important cause of cirrhosis and hepatocellular carcinoma. Its pathological alterations primarily include steatosis, lobular inflammation, and hepatocyte ballooning, eventually leading to the development of liver fibrosis and even hepatic cancer[1]. Approximately one-third of patients with MAFLD develop metabolic associated steatohepatitis (MASH). MAFLD is the result of multiple factors, including insulin resistance (IR), chronic oxidative stress, and lipotoxicity, as well as other conditions that promote hepatic inflammation and fibrosis[2-4]. Despite remarkable progress in understanding the molecular pathogenesis of $\mathrm{MASH}$, to date, no effective drugs exist for its treatment. Therefore, it is vital to investigate the molecular mechanisms underlying MAFLD to ensure prevention and proper treatment. 
Nuclear factor erythroid 2 related factor 2 (Nrf2) is a key transcription factor that regulates intracellular redox homeostasis[2]. Previous studies have shown that a reduction or enhancement in the activity of Nrf2 does not prevent diet-induced obesity and has less effect on lipid metabolism[5]. However, it has also been found that pharmacological activation of Nrf2 inhibits hepatic steatosis and reduces MASH and hepatic fibrosis in obese mice[6]. In addition, Nrf2 activation has been reported to inhibit hepatosteatosis and MASH on knockdown of its repressor Kelch-like ECH-associated protein-1 in methionine- and choline-deficient diet-fed mice[7]. Therefore, at present, the role of Nrf2 in the pathogenesis of MAFLD is contradictory, and accordingly, further studies are warranted.

Autophagy is a cellular process that degrades intracellular organelles and proteins. It targets invading microorganisms and involves abnormal protein accumulation to lysosomes degrade[8]. Functionally, autophagy is necessary to maintain the stability of the intracellular environment, and defects in autophagy are evidently related to the pathological process of some human diseases[9]. Previous studies have found that autophagy breaks down triglycerides (TG) via lipophagy and that the inhibition of autophagy in hepatocytes can cause hepatic lipid accumulation $[10,11]$. In addition, genetic or pharmacological activation of autophagy has been found to reduce hepatosteatosis[12, 13]. However, the underlying mechanism via which autophagy regulates hepatic lipid accumulation remains debatable.

Some proteins play a pivotal role in the initiation of autophagy; for example, Atg3 and Atg7 downregulation is reportedly related to autophagosomes formation[14]. Further, Atg7 overexpression has been found to alleviate MAFLD and IR in ob/ob mice and high-fat diet (HFD)-fed mice[15]. Some studies have also shown that the late stage of autophagy (such as autophagosome-lysosome fusion) plays a fundamental role in MAFLD occurrence and development, rather than in the initiation of autophagy[16]. In recent years, Nrf2 has been reported to regulate autophagy initiation through the signaling adapter p62dependent pathway in response to oxidative damage via a positive feedback loop[17]. However, it remains unknown whether Nrf2 affects MAFLD occurrence and development by regulating autophagy in the liver.

Herein we established an MAFLD model in HFD-fed Nrf2 KO mice; further, a cell model of lipid accumulation was established using mouse primary hepatocytes (MPHs) treated with free fatty acids (FAs). Using these models, we investigated the relationship between Nrf2 and autophagy and its role in the development of MAFLD.

\section{Materials And Methods}

Mice and human hepatic tissue studies

Male 8-week-old C57BL/6J mice (wild-type, WT) were purchased from the Experimental Animal Center (Chongqing Medical University). 8-week-old male Nrf2 KO mice were purchased from Shanghai Bio-model and Ganismsci \& Tech Develop Co., Ltd. (Shanghai, China). SREBP-1c knockout (SREBP-1c KO) mice were generously provided by Dr. Guan (Advanced Institute for Medical Sciences, Dalian Medical University, Liaoning, China). Nrf2 KO and SREBP-1c KO mice were generated as previously reported[18]. 
To establish MAFLD model induced by HFD, mice were fed a chow diet (NCD; $10 \%$ fat) or an HFD (60\% fat; D12492; New Brunswick, NJ 08901 USA) for 12 weeks. Subsequently, mice were sacrificed and tissues were collected for further analyses. All animal protocols were approved by the Chongqing Medical University Animal Studies Committee and were consistent with the National Institutes of Health Guide for the Care and Use of Laboratory Animals (NIH Publications No. 8023, revised 1978).

Liver tissues were obtained via hepatic biopsy from six patients with MAFLD and six liver transplantation donors from the Department of Surgery, Xinqiao Hospital, Third Military Medical University. The protocols were reviewed and supported by the Ethics Committee of Xinqiao Hospital, Third Military Medical University, Chongqing China.

Gene Set Enrichment Analysis (GSEA)

Thirty-nine MAFLD samples from GSE89632 were divided into high and low expression groups, according to the median expression level of Nrf2. GSEA was then performed using GSEA v4.1.0 platform (http://software.broadinstitute.org/gsea/index.jsp)[19]. Gene ontology (GO) and Kyoto Encyclopedia of Genes and Genomes (KEGG) gene sets downloaded from the Molecular Signature Database served as reference. $P<0.05$ was used for the identification of statistically significant GO and KEGG terms.

Biological Process (BP) Enrichment Analysis

Based on the GPL4134 platform, the(GSE35124)dataset was downloaded from the Gene Expression Omnibus (GEO) database. The raw expression data were preprocessed by the RMA algorithm in the affy package using the $\mathrm{R}$ statistical software. Differentially expressed genes (DEGs) were identified with the limma package[20]. BP enrichment analysis was performed with DAVID v6.8[21]. $P<0.05$ determined statistical significance.

Cell culture and treatment

MPHs were isolated from 8-week-old WT, Nrf2 KO, and SREBP-1c KO mice, as previously described[18], and were cultured in DMEM/F12. HepG2 cells were cultured in DMEM with $10 \%$ FBS. On reaching $70 \%$ confluence, they were incubated with FA mixture (FAs, $1 \mathrm{mmol} / \mathrm{L})$ at a 2:1 ratio of oleate/palmitate or BSA for $24 \mathrm{~h}$, then treated with DMSO or ML385, an Nrf2 inhibitor, for $12 \mathrm{~h}$ (5uM, MCE, Shanghai, China).

Hematoxylin-Eosin (H\&E) and Oil Red O staining

Paraffin-embedded livers from mice were processed, and paraffin sections ( 5 um) were stained with H\&E. Frozen hepatic sections, HepG2 cells and MPHs were stained with $0.3 \%$ Oil Red 0 by standard procedures, as reported[22].

Immunohistochemistry (IHC)

Mice were anesthetized and perfused with $4 \%$ paraformaldehyde for $10 \mathrm{~min}$. The liver was excised, transferred to $4 \%$ paraformaldehyde, and incubated at $4^{\circ} \mathrm{C}$ for 1 day. The samples were embedded by 
paraffin and sliced. IHC to assess the expression levels of Nrf2, p62/SQSTM1 (p62), and LC3II were engaged as previously publication[23]. The primary antibodies used for IHC were anti-Nrf2, anti-p62, and anti-LC3 (1:400; ab62352, ab91526, ab229327; Abcam, Cambridge, England).

Autophagy flux measurement using adenovirus expressing mRFP-GFP-LC3 (Ad-mRFP-GFP -LC3)

HepG2 cells and MPHs from WT and Nrf2 KO mice were infected with Ad-mRFP-GFP-LC3 (HanBio, Wuhan, China) as manufacturer's instructions. The confocal microscopy was used for observing the morphology of LC3 positive autophagosomes and autolysosomes. And the cells expressing LC3 were labeled with mRFP-GFP. Autophagosomes thus appeared as a yellow spot $\left(\mathrm{RFP}^{+} \mathrm{GFP}^{+}\right)$, while autolysosomes appeared as a red spot $\left(\mathrm{RFP}^{+} \mathrm{GFP}^{-}\right)$. The accumulation of LC3 spots was quantified to evaluate autophagy flux[24].

Determination of hepatic TG contents

Hepatic TG content was measured using a commercial kit (Applygen Technologies, Beijing, China) according to manufacturer instructions[25].

quantitative real-time PCR (qRT-PCR) and Western blotting

qRT-PCR was performed as previously reported[26]. Table S1 lists the forward and reverse primer sequences used for qRT-PCR, and Table S2 lists the primary antibodies used for Western blotting. qRTPCR and Western blotting results were calibrated to $\beta$-actin or GAPDH[25].

Biochemical analysis

Serum total cholesterol (TC) and TG were enzymatically measured using an auto-analyzer. Serum alanine transaminase (ALT) and aspartate transaminase (AST) were measured using a commercial kit (Kebo biological industry co. LTD, Shandong, China) according to manufacturer instructions.

Luciferase reporter assay

Genomic PCR was performed to obtain DNA fragments containing the full-length mouse LAMP1 promoter and all deletion constructs $(-1740 / 0,-1019 / 0$, and $-851 / 0 \mathrm{bp})$ were generated as previously described[25]. Purified PCR products and pGL3-basic luciferase reporter vector (Promega, Madison, WI, USA) were digested with Xhol and HindIII. Recombinant plasmids (pGL3-LAMP1-Luc) were verified by restriction endonuclease digestion and DNA sequencing. The primers used for the DNA fragments were listed in Table S3. Luciferase reporter assay was performed using a previously reported standard protocol[18]. Briefly, MPHs from WT and Nrf2 KO mice were transfected with reporter and Renilla-Luc plasmids (Promega, Madison, WI, USA) using Lipofectamine 3000 (Life Technologies). Luciferase activities were examined by a Dual luciferase kit (Promega) at $48 \mathrm{~h}$ post-transfection[25].

ChIP-qPCR 
ChIP experiments were performed using MPHs of WT mice[27]. Briefly, 1\% formaldehyde (EM grade; Thermo-Scientific) was used for crosslinking, a cell Lysis Buffer was used to lyse the cells, and ultrasound treatment was used to fragment DNA. The chromatin fraction was incubated with anti-Nrf2 (ab62352, Abcam, Cambridge, USA) or unspecific IgG (sc-2027, Santa Cruz Biotechnology Inc.). qPCR was conducted using SYBR Green Mix (Takara Bio, Otsu, Japan) for DNA fragment analysis. The amplicons were separated by electrophoresis on an agarose gel containing ethidium bromide and visualized under UV light. Specific primers were listed Table S4.

Statistics

The data were presented as the mean \pm SEM. Statistical analysis was performed via Microsoft Excel and Prism software (Graphpad, La Jolla, CA). A two-way ANOVA with a least significant difference post-hoc test was used to compare the mean values between multiple groups, and an unpaired two-sided Student $t$-test was used for comparisons between two groups. $p<0.05$ was considered statistically significant.

\section{Results}

\section{Upregulation of hepatic Nrf2 in obese mice and patients with MAFLD}

To determine the role of Nrf2 in obesity-related MAFLD, we first assessed the expression levels of Nrf2 and lipogenesis-related genes in patients with MAFLD. We observed that the expression levels of lipogenesis-related genes, including C/EBPa and $B$, Cidea and Ly6D, were significantly upregulated, suggestive of an increase in lipid synthesis in the liver (Fig. S1A). Importantly, the expression of Nrf2 at both mRNA and protein was markedly upregulated in the liver of patients with MAFLD, indicating its involvement in hepatic lipid deposition (Fig. 1A, B).

To further determine the role of Nrf2 in hepatosteatosis, we investigated hepatic Nrf2 expression levels in WT mice fed either NCD or HFD. As indicated in Fig. S1 B-D, histological and biochemical parameters confirmed MAFLD occurrence in HFD-fed mice. Next, we performed IHC, qRT-PCR and Western blots to investigate hepatic expression levels of Nrf2 mRNA and protein in WT mice fed either NCD or HFD, and we found that Nrf2 expression was markedly elevated both at mRNA and protein levels in HFD-fed mice (Fig. 1C, D). IHC also revealed that Nrf2 expression level was up-regulated in the liver from HFD-fed mice (Fig. 1E). Consistent with the results in mice and humans, the mRNA and protein expression levels of Nrf2 in FAs-treated MPHs were significantly up-regulated as compared to those in PBS-treated MPHs (Fig. 1F, G). These data demonstrate a key role of Nrf2 in MAFLD.

\section{Deletion of Nrf2 facilitates hepatosteatosis induced by HFD}

Considering the changes in Nrf2 mRNA and protein expression levels observed in obese mice and patients with MAFLD, we generated Nrf2 KO mice, and 8-week-old male WT and Nrf2 KO mice were fed either NCD or HFD to investigate the role of Nrf2 in hepatosteatosis (Fig. 2A). No significant changes were 
observed in body weight and hepatic weight between NCD-fed Nrf2 KO and WT mice (Fig. 2B, D, E); however, HFD-fed Nrf2 KO mice showed significantly higher body weight and hepatic weight as compared with WT mice (Fig. 2B, D, E). Furthermore, at the macroscopic level, the liver showed a white appearance in HFD-fed Nrf2 KO mice (Fig. 2C). HE and Oil Red $\mathrm{O}$ staining revealed higher lipid accumulation in the liver of HFD-fed Nrf2 KO mice (Fig. 2F, G). Consistent with these changes, hepatic TG content, serum TG, $\mathrm{CHO}, \mathrm{ALT}$, and AST concentrations in Nrf2 KO mice fed an HFD were significantly increased than those in WT mice fed with the same diet (Fig. 2H-L). These data collectively suggest that hepatic Nrf2 has an important role in HFD-induced hepatosteatosis.

\section{Deletion of Nrf2 enhances the expression of lipogenesis- related genes}

To gain insights into the mechanism underlying the regulation of hepatic lipid metabolism by Nrf2, we analyzed genomic data pertaining to the liver tissues of patients with MAFLD from the GSE89632 dataset. GSEA revealed that Nrf2-related genes were mainly enriched in lipid metabolism (Fig. 3A). In addition, BP analysis of DEGs (GSE35124) revealed that Nrf2-related genes were most enriched in fatty acid synthesis (Fig. 3B).

To verify the relationship between Nrf2 and lipid metabolism, we investigated the expression levels of lipid-related genes in the liver of WT and Nrf2 KO mice fed either NCD or HFD. We observed that in comparison with HFD-fed WT mice, the protein expression levels of lipogenesis-related genes, including FAS, SCD-1, and Sterol regulatory element-binding protein 1c (SREBP-1c), were significantly upregulated in HFD-fed Nrf2 KO mice, while the phosphorylation level of ACC was significantly decreased (Fig. 3C). Further, oil red $\mathrm{O}$ staining showed that the lipid droplet contents of FAs-treated MPHs from Nrf2 KO mice and HepG2 cells treated with ML385 were significantly increased compared with those from WT mice and treated with DMSO (Fig. 3D, E). TG contents of MPHs from Nrf2 KO mice and HepG2 cells treated with ML385 were also higher (Fig. 3F, G). In these MPHs and HepG2 cells, the protein expression levels of lipogenesis-related genes, including SCD-1, FAS, and SREBP-1C, were significantly upregulated relative to control MPHs and HepG2 cells, while the phosphorylation level of ACC was significantly lower (Fig. $3 \mathrm{H}, \mathrm{I})$. Therefore, the results of our bioinformatics analyses and in vivo and in vitro studies validated that Nrf2 deficiency promotes lipogenesis and hepatic lipid accumulation.

\section{SREBP-1 is crucial for Nrf2 to regulate adipogenesis}

To further investigate how Nrf2 affects lipid metabolism, SREBP-1 KO mice were generated and MPHs were isolated, they were then incubated with BSA or FAs and treated with ML385 (an Nrf2 inhibitor) or DMSO (Fig. 4A). Further, the contents of TG and lipid droplets were significantly decreased in SREBP-1KO mice treated with ML385 and FAs compared with WT mice treated ML385 and FAs, but still higher than those in SREBP-1KO mice treated with DMSO and FAs (Fig. 4B, C). Importantly, the mRNA expression level of FAS and SCD-1 in ML385-treated MPHs from WT mice was significantly higher than that in DMSO- 
treated MPHs, but no significant changes were observed in MPHs from SREBP-1 KO mice (Fig. 4D, E). These data confirmed that SREBP-1 plays a major role in Nrf2-mediated lipogenesis.

\section{Hepatic autophagy signaling is involved in hepatosteatosis regulation by $\mathrm{Nrf} 2$}

Autophagy is reportedly involved in lipid metabolism. To further investigate the mechanism by which Nrf2 mediates hepatic lipid metabolism, we performed GSEA using the liver tissues of patients with MAFLD from the GSE89632 dataset. We found that Nrf2-related genes were mainly enriched in the pathway related to lysosome function (Fig. 5A). Next, to confirm the findings of bioinformatics analysis, we examined the expression levels of LC3II (microtubule-associated protein 2 light chain 3 ) and SQSTM1/p62 in the liver of Nrf2 KO and WT mice fed either NCD or HFD[28]. The protein expression level of LC3II in the liver of HFD-fed Nrf2 KO mice was significantly downregulated in comparison with that in WT mice, which caused a significant decrease in the LC3II/LC3I ratio (Fig. 5B), suggesting an inhibition of autophagic flux. Furthermore, the protein expression of Atg7 in the liver of HFD-fed Nrf2 KO mice was significantly down-regulated in comparison with that in WT mice, while Atg3 was unchanged (Fig. 5B). Interestingly, we found that the protein expression level of SQSTM1/p62 (a substrate of autophagy) was markedly downregulated in the liver of Nrf2 KO mice than in that of WT mice (Fig. 5B). We speculate that the down-regulation of p62 may be due to the effect of Nrf2 knockout on p62 transcription as previous publications.[29] Moreover, in the liver of Nrf2 KO mice, IHC staining showed decreased expression levels of LC3II and SQSTM1/p62 (Fig. 5C). These results indicated that the lack of Nrf2 inhibited SQSTM1/p62 as well as autophagic flux.

Lysosomal degradation plays an important role in maintaining autophagic flux and recycling hepatic lipid droplets. We thus evaluated the expression levels of LAMP1 and LAMP2, which are lysosomal-associated membrane proteins,[30] in the liver of Nrf2 KO and WT mice. The protein expression level of LAMP1 was significantly downregulated in HFD-fed Nrf2 KO mice relative to that in WT mice, but the expression level of LAMP2 did not change (Fig. 5D), suggesting that Nrf2 deficiency causes lysosomal membrane dysfunction.

\section{Effects of Nrf2 deficiency on lipid accumulation and autophagy in vitro}

To further verify the relationship between Nrf2 deficiency and autophagy at the cellular level, MPHs from WT and Nrf2 KO mice were treated with FAs. As with in vivo studies, Western blotting showed that p62 protein expression level and LC3II densitometry in FAs-treated MPHs from Nrf2 KO mice and HepG2 cells treated with ML385 were significantly decreased compared with those in MPHs from WT mice and HepG2 cells treated with DMSO (Fig. 6A, C). LAMP1 and Atg7 protein levels also were lower in MPHs from Nrf2 KO mice and HepG2 cells treated with ML385 (Fig. 6B, C). To observe the effect of Nrf2 on autophagic flux more intuitively, we used a tandem labeled mRFP-GFP-LC3 reporter. HepG2 cells and MPHs from WT and Nrf2 KO mice were infected with Ad-mRFP-GFP-LC3 and LC3 punctate numbers were quantified for assessing the effect of Nrf2 inhibition or deficiency on autophagic flux. FAs-treated HepG2 
cells and MPHs showed increased yellow fluorescent puncta and decreased red fluorescent puncta after $12 \mathrm{~h}$ exposure. Cells from Nrf2 KO mice or treated with ML385 showed an even more significant reduction in red punctate $\left(\mathrm{GFP}^{-}\right.$and $\left.\mathrm{mRFP}^{+}\right)$, compared with MPHs from WT mice and HepG2 cells treated with DMSO, indicating that Nrf2 depletion further inhibited autophagic flux induced by FAs (Fig. 6D).

\section{Nrf2 inhibits autophagy via the transcriptional regulation of LAMP1 promoter}

One previous study has predicted LAMP1 region contains a putative ARE sequence.[31] To determine how Nrf2 regulates LAMP1 expression, we investigated the effect of Nrf2 on LAMP1 transcription in MPHs from WT mice. Figure 7A shows a diagram for predicting the binding site of Nrf2 and LAMP1 promoter. ChIP-qPCR results confirme that Nrf2 directly binds to the -1740 bp to -1726 bp region of LAMP1 promoter (Fig. 7B, C). Luciferase reporter assay results further indicated that a potential Nrf2-regulating site for LAMP1 promoter was located -1740 bp to -1726 bp upstream of the transcriptional start site (Fig. 7D).

\section{Discussion}

The pathogenesis of MAFLD is considered to be a complex, multifactorial process, and autophagy, chronic inflammation, and mitochondrial dysfunction are evidently involved in its occurrence and development[32]. Although Nrf2 has been reported to be involved in the pathogenesis of MAFLD, the results reported by previous studies are contradictory $[5,6,33]$. Therefore, the exact mechanism of Nrf2 in the occurrence and development of MAFLD remains unknown. Previous studies focused on the antioxidant effect of Nrf2 on lipid accumulation in the liver, while there were few reports about the Nrf2 regulation of lipogenesis in the liver[34]. In addition, previous studies were mostly descriptive and observational studies, lacking in-depth research, especially the molecular mechanism. In view of the particularity and complexity of MAFLD, it is urgent to further study the role of Nrf2 in the pathogenesis of MAFLD. Herein 1) our data confirmed that hepatic Nrf2 expression level was upregulated in patients with MAFLD and obese mice; 2) Nrf2 deficiency was observed to promote the development of hepatic lipid deposition when mice were fed HFD; 3) SREBP-1 activation was identified to be crucial for Nrf2 deficiency to promote fatty acid synthesis; 4) Nrf2 deficiency was found inhibit lipolysis by weakening autophagy; and finally, 5) Nrf2 deficiency was found to attenuate autophagy by inhibiting the transcription of LAMP1 promoter. Our findings thus reveal, in detail, the molecular mechanisms by which Nrf2 regulates hepatic lipid metabolism.

Herein our findings further confirmed that Nrf2 deficiency aggravated HFD-induced hepatosteatosis and FAs-induced intracellular lipid deposition. Consistent with our data, some previous studies have also found that Nrf2 deletion in hepatocytes resulted in a worse metabolic phenotype[33], resulting in higher liver tissue weight and the expression of inflammation-related factors in the liver[35]. In addition, a study reported that administering agonists activated Nrf2 to prevent obesity-induced by HFD in mice[36]. In contrast, another study reported that the lack of Nrf2 conferred protection from HFD-induced obesity in 
mice[5]. Further, in genetically modified mice, Nrf2 has been observed to neither prevent the development of HFD-induced obesity nor show any involvement in the direct regulation of hepatic lipid metabolism[5, 37]. These contradictory results could be attributed to, for example, differences in experimental design, sources of fat in the diet, time of feeding the diet (i.e., HFD), gender, genetic background, and age of mice. Consequently, the mechanism by which Nrf2 deficiency leads to hepatic lipid accumulation is not completely understood. In this study, the genetic model was established using Nrf2 KO C57BL/6J mice to avoid the off-target effects of chemicals. All the mice were male, and to mimic the human diet, we fed the animals with $60 \%$ HFD or NCD for 12 weeks. Our data from patients with MAFLD further confirmed the relationship between Nrf2 and hepatosteatosis. Therefore, by controlling these factors, we believe that Nrf2 deficiency promotes diet-induced hepatic lipid deposition and obesity. Nevertheless, further studies are warranted to investigate the role of Nrf2 in hepatic lipid metabolism in more detail and to determine if Nrf2 interacts with other pathways that may lead to hepatic lipid accumulation.

SREBP-1, a transcription factor, regulates adipogenesis and glucose utilization in the liver, playing a pivotal role in fatty acid synthesis[18, 38]. In vivo and in vitro experiment results revealed that Nrf2 deficiency-mediated lipid accumulation was associated with significantly upregulated expression levels of genes encoding SREBP-1, FAS, and SCD-1. However, in MPHs from SREBP-1 KO mice, Nrf2 deficiency significantly reduced lipid accumulation. Thus, SREBP-1 appears to play a key role in Nrf2-mediated regulation of lipid metabolism. These results are consistent with previous studies[34, 36]. In addition, a previous study involving adipocytes reported that Nrf2 recruited SREBP-1 promoter and enhanced its transcription[39]. Importantly, we used SREBP-1 KO mice to provide unequivocal genetic evidence that Nrf2 can play a protective role in the initiation of hepatic lipid accumulation.

To obtain further insights into whether Nrf2 regulates lipid metabolism via multiple pathways, we performed GSEA using data from the GSE89632 database and found that Nrf2-related genes were mainly enriched in the pathway related to "Iysosome." It is well known that autophagy decomposes intracellular lipids through lysosomal degradation[40]. Therefore, autophagy may be a candidate target for the Nrf2 regulation of hepatic lipid metabolism. Autophagy was recently reported to be associated with fat metabolism[41]. Autophagy dysfunction in the liver can inhibit fatty acid $\beta$-oxidation and TG output, causing hepatosteatosis in vivo[14, 42]. When autophagy is stimulated in the liver, hepatosteatosis improves[43]. In recent years, there are some lines of evidence indicating that Nrf2 is associated with autophagy in lung cancer cells and skeletal muscle[44-46]. In addition, Tang et al. reported that Nrf2 elevated the expression levels of genes related to autophagy in the brain of mice with age[47]. In contrast, Kitaoka et al. found that Nrf2 deficiency did not affect autophagy in the skeletal muscle of denervated mice[48]. We speculate that these contradictory results may be due to the different mechanisms of Nrf2 in different tissues and cells. Therefore, in the present study, we used Nrf2 KO mouse liver and primary hepatocytes to further explore the relationship between Nrf2 and autophagy. We found that in comparison with HFD-fed WT mice, autophagy was weakened in HFD-fed Nrf2 KO mice, as manifested by decreased LC3II/LC3I ratio and reduced p62, LAMP1, and Atg7 expression levels. Thus, our findings indicate that Nrf2 has a positive regulatory effect on HFD-induced autophagy in vivo. Although these 
results are consistent with a previous study[47] but different from those of others[46, 48], we believe that Nrf2 regulates hepatosteatosis via autophagy.

Autophagosomes mature by fusing with lysosomes to form autophagolysosomes, where they are degraded. We evaluated the effect of Nrf2 on the fusion of lysosomes with autophagosomes, which was mediated by the lysosomal proteins LAMP1 and LAMP2[49], and found that Nrf2 deficiency-mediated LAMP1 inhibition impacted the fusion of autophagosomes and lysosomes in hepatocytes, resulting in the impairment of the autophagic degradation capacity of lysosomes and leading to hepatic lipid accumulation. To better understand the molecular mechanism by which Nrf2 deficiency inhibits LAMP1 expression, we performed ChIP-qPCR and dual luciferase assay, and the obtained findings confirmed that Nrf2 binds to LAMP1 promoter and regulates its transcriptional activity. A potential Nrf2-regulating site for the LAMP1 promoter was located at -1740 to -1726 bp upstream of the transcriptional start site.

As with other studies, even our study has some limitations: 1) we did not include Nrf2 hepatic-specific KO mice, mainly because they may still show low expression levels of Nrf2 in the liver, with the expression levels being potentially higher in other tissues (compensatory increase)[50], and 2) the potential mechanism for the interaction of Nrf2 with Atg7 remains to be determined.

\section{Conclusions}

To conclude, our data further reveal that Nrf2 deficiency promoted hepatosteatosis induced by HFD. Importantly, our study elucidated novel molecular mechanisms of Nfr2 regulating lipid metabolism in the liver through bioinformatics analysis and molecular biology technology, that is, Nrf2 can inhibit hepatic lipid accumulation by promoting autophagy through regulating LAMP1 transcriptional activity. Furthermore, we also provide genetic evidence for the interaction between Nrf2 and SREBP-1 (Fig. S2). Therefore, using an Nrf2 activator to promote the formation of autophagosomes and lysosomal acidification seems to be a promising strategy to treat MAFLD.

\section{Abbreviations}

ALT: Serum alanine transaminase

AST: aspartate transaminase

FAs: free fatty acids

HFD: high fat diet

MAFLD: metabolic associated fatty liver disease

MASH: metabolic associated steatohepatitis

MPHs: mouse primary hepatocytes 
Nrf2: Nuclear factor erythroid 2-related factor 2

Nrf2 KO: Nrf2 knockout

NCD: chow diet

TG: triglycerides

TC: Serum total cholesterol

WT: C57BL/6J mice

\section{Declarations}

\section{Ethics approval and consent to participate}

This study was approved by Chongqing Medical University Animal Studies Committee and the methods were carried out in accordance with the approved guidelines.

\section{Consent for publication}

Not applicable.

\section{Availability of data and materials}

All data generated or analyzed during this study are included in this published article.

\section{Competing interests}

No potential conflicts of interest relevant to this article were reported.

\section{Funding}

This study was supported by Grants from the National Natural Science Foundation of China (No. 81470045 and 81670755); Research Institute performance incentive and guidance project of Chongqing Science and Technology Bureau (cstc2019jxj|130006) and Chongqing Science and Technology Bureau and Health Commission Joint TCM technology innovation and application development project (2020zy013540).

\section{Author Contributions.}

S.Q., Z.L., M.W., M.Y. and Q.W. researched and analyzed the data. C.C. and Z.M. contributed to the writing of the manuscript and helpful discussion. H.Z. provided research material and directed the project and contributed to the discussion. L. L. and G. Y. wrote and edited the manuscript. L. L. and G. Y. were the guarantors of this work and, as such, had full access to all the data in the study and takes responsibility for the integrity of the data and the accuracy of the data analysis. 
Acknowledgments:

We are grateful to Dr. Guan for donating us SREBP-1c KO mice.

Author information

Sheng Qiu, Zerong Liang and Qinan Wu have contributed equally to this work.

Affiliations

Department of Endocrinology, the 2st Affiliated Hospital, Chongqing Medical University, Chongqing, China.

Sheng Qiu, Zerong Liang, Miao Wang, Mengliu Yang \& Gangyi Yang

Key Laboratory of Diagnostic Medicine (Ministry of Education) and Department of Clinical Biochemistry, College of Laboratory Medicine, Chongqing Medical University

Zerong Liang \& Ling Li

Da Zu Affiliated Hospital, Chongqing Medical University, Chongqing, China.

Qinan Wu

Endocrinology, SBMS, Faculty of Medicine, University of Queensland, Brisbane, 4072. Australia

Chen Chen

Department of Endocrinology, Xinqiao Hospital, Third Military Medical University, Chongqing, China Hongting zheng

Department of Hypertension and Endocrinology, Daping Hospital, Third Military Medical University, Chongqing Institute of Hypertension, Chongqing, China

Zhiming Zhu

\section{References}

1. Anty R, and Gual P. [Pathogenesis of non-alcoholic fatty liver disease]. Presse Med. 2019;48(12):1468-1483. Http://dx.doi.org/10.1016/j.lpm.2019.09.051

2. Colca J. NASH (nonalcoholic steatohepatitis), diabetes, and macrovascular disease: multiple chronic conditions and a potential treatment at the metabolic root. Expert Opin Investig Drugs. 2020;29(2):191-196. Http://dx.doi.org/10.1080/13543784.2020.1715940 
3. Kim YN, Shin JH, Kyeong DS, Cho SY, Kim MY, Lim HJ, et al. Ahnak deficiency attenuates high-fat diet-induced fatty liver in mice through FGF21 induction. Exp Mol Med. 2021;53(3):468-

482. Http://dx.doi.org/10.1038/s12276-021-00573-3

4. Liu XL, Ming YN, Zhang JY, Chen XY, Zeng MD, and Mao YM. Gene-metabolite network analysis in different nonalcoholic fatty liver disease phenotypes. Exp Mol Med.

2017;49(1):e283. Http://dx.doi.org/10.1038/emm.2016.123

5. Zhang YK, Wu KC, Liu J, and Klaassen CD. Nrf2 deficiency improves glucose tolerance in mice fed a high-fat diet. Toxicol Appl Pharmacol. 2012;264(3):305-

314. Http://dx.doi.org/10.1016/j.taap.2012.09.014

6. Xu L, Nagata N, and Ota T. Impact of Glucoraphanin-Mediated Activation of Nrf2 on Non-Alcoholic Fatty Liver Disease with a Focus on Mitochondrial Dysfunction. Int J Mol Sci.

2019;20(23)Http://dx.doi.org/10.3390/ijms20235920

7. Ramadori P, Drescher H, Erschfeld S, Schumacher F, Berger C, Fragoulis A, et al. Hepatocytespecific Keap1 deletion reduces liver steatosis but not inflammation during non-alcoholic steatohepatitis development. Free Radic Biol Med. 2016;91:114-

126. Http://dx.doi.org/10.1016/j.freeradbiomed.2015.12.014

8. Codogno P, and Meijer AJ. Autophagy and signaling: their role in cell survival and cell death. Cell Death Differ. 2005;12 Suppl 2:1509-1518. Http://dx.doi.org/10.1038/sj.cdd.4401751

9. Mialet-Perez $\mathrm{J}$, and Vindis C. Autophagy in health and disease: focus on the cardiovascular system. Essays Biochem. 2017;61(6):721-732. Http://dx.doi.org/10.1042/ebc20170022

10. Huda N, Zou H, Yan S, Khambu B, and Yin XM. Analysis of Autophagy for Liver Pathogenesis. Methods Mol Biol. 2019;1880:481-489. Http://dx.doi.org/10.1007/978-1-4939-8873-0_30

11. Khambu B, Yan S, Huda N, Liu G, and Yin XM. Autophagy in non-alcoholic fatty liver disease and alcoholic liver disease. Liver Res. 2018;2(3):112-119. Http://dx.doi.org/10.1016/j.livres.2018.09.004

12. Ren $H$, Wang $D$, Zhang $L$, Kang X, Li Y, Zhou X, et al. Catalpol induces autophagy and attenuates liver steatosis in ob/ob and high-fat diet-induced obese mice. Aging (Albany NY). 2019;11(21):94619477. Http://dx.doi.org/10.18632/aging.102396

13. Zhou T, Chang L, Luo Y, Zhou Y, and Zhang J. Mst1 inhibition attenuates non-alcoholic fatty liver disease via reversing Parkin-related mitophagy. Redox Biol. 2019;21:101120. Http://dx.doi.org/10.1016/j.redox.2019.101120

14. Martinez-Lopez N, and Singh R. Autophagy and Lipid Droplets in the Liver. Annu Rev Nutr. 2015;35:215-237. Http://dx.doi.org/10.1146/annurev-nutr-071813-105336 
15. Yang L, Li P, Fu S, Calay ES, and Hotamisligil GS. Defective hepatic autophagy in obesity promotes ER stress and causes insulin resistance. Cell Metab. 2010;11(6):467-

478. Http://dx.doi.org/10.1016/j.cmet.2010.04.005

16. Park HW, Park H, Semple IA, Jang I, Ro SH, Kim M, et al. Pharmacological correction of obesityinduced autophagy arrest using calcium channel blockers. Nat Commun.

2014;5:4834. Http://dx.doi.org/10.1038/ncomms5834

17. Ghosh S, Choudhury S, Chowdhury O, Mukherjee S, Das A, Sain A, et al. Inflammation-induced behavioral changes is driven by alterations in Nrf2-dependent apoptosis and autophagy in mouse hippocampus: Role of fluoxetine. Cell Signal.

2020;68:109521. Http://dx.doi.org/10.1016/j.cellsig.2019.109521

18. Yang M, Liang Z, Yang M, Jia Y, Yang G, He Y, et al. Role of bone morphogenetic protein-9 in the regulation of glucose and lipid metabolism. Faseb j. 2019;33(9):10077-

10088. Http://dx.doi.org/10.1096/fj.201802544RR

19. Subramanian A, Tamayo P, Mootha VK, Mukherjee S, Ebert BL, Gillette MA, et al. Gene set enrichment analysis: a knowledge-based approach for interpreting genome-wide expression profiles. Proc Natl Acad Sci U S A. 2005;102(43):15545-15550. Http://dx.doi.org/10.1073/pnas.0506580102

20. Ritchie ME, Phipson B, Wu D, Hu Y, Law CW, Shi W, et al. limma powers differential expression analyses for RNA-sequencing and microarray studies. Nucleic Acids Res.

2015;43(7):e47. Http://dx.doi.org/10.1093/nar/gkv007

21. Huang da W, Sherman BT, and Lempicki RA. Bioinformatics enrichment tools: paths toward the comprehensive functional analysis of large gene lists. Nucleic Acids Res. 2009;37(1):1-

13. Http://dx.doi.org/10.1093/nar/gkn923

22. Fu J, Li C, Zhang G, Tong X, Zhang H, Ding J, et al. Crucial Roles of 5-HT and 5-HT2 Receptor in Diabetes-Related Lipid Accumulation and Pro-Inflammatory Cytokine Generation in Hepatocytes. Cell Physiol Biochem. 2018;48(6):2409-2428. Http://dx.doi.org/10.1159/000492656

23. Wang X, Zhang X, Chu ESH, Chen X, Kang W, Wu F, et al. Defective lysosomal clearance of autophagosomes and its clinical implications in nonalcoholic steatohepatitis. Faseb j. 2018;32(1):3751. Http://dx.doi.org/10.1096/fj.201601393R

24. Yu T, Guo F, Yu Y, Sun T, Ma D, Han J, et al. Fusobacterium nucleatum Promotes Chemoresistance to Colorectal Cancer by Modulating Autophagy. Cell. 2017;170(3):548-

563.e516. Http://dx.doi.org/10.1016/j.cell.2017.07.008

25. Zhang C, Luo X, Chen J, Zhou B, Yang M, Liu R, et al. Osteoprotegerin Promotes Liver Steatosis by Targeting the ERK-PPAR-y-CD36 Pathway. Diabetes. 2019;68(10):1902- 
26. Zhou $M, X u X$, Wang $H$, Yang $G$, Yang $M$, Zhao X, et al. Effect of central JAZF1 on glucose production is regulated by the PI3K-Akt-AMPK pathway. Faseb j. 2020;34(5):7058-

7074. Http://dx.doi.org/10.1096/fj.201901836RR

27. Cui A, Fan H, Zhang Y, Zhang Y, Niu D, Liu S, et al. Dexamethasone-induced Krüppel-like factor 9 expression promotes hepatic gluconeogenesis and hyperglycemia. J Clin Invest. 2019;129(6):22662278. Http://dx.doi.org/10.1172/jci66062

28. Zhang X, Wu WK, Xu W, Man K, Wang X, Han J, et al. C-X-C Motif Chemokine 10 Impairs Autophagy and Autolysosome Formation in Non-alcoholic Steatohepatitis. Theranostics. 2017;7(11):28222836. Http://dx.doi.org/10.7150/thno.19068

29. Jain A, Lamark T, Sjøttem E, Larsen KB, Awuh JA, Øvervatn A, et al. p62/SQSTM1 is a target gene for transcription factor NRF2 and creates a positive feedback loop by inducing antioxidant response element-driven gene transcription. J Biol Chem. 2010;285(29):2257622591. Http://dx.doi.org/10.1074/jbc.M110.118976

30. Babuta M, Furi I, Bala S, Bukong TN, Lowe P, Catalano D, et al. Dysregulated Autophagy and Lysosome Function Are Linked to Exosome Production by Micro-RNA 155 in Alcoholic Liver Disease. Hepatology. 2019;70(6):2123-2141. Http://dx.doi.org/10.1002/hep.30766

31. Pajares M, Jiménez-Moreno N, García-Yagüe Á J, Escoll M, de Ceballos ML, Van Leuven F, et al. Transcription factor NFE2L2/NRF2 is a regulator of macroautophagy genes. Autophagy. 2016;12(10):1902-1916. Http://dx.doi.org/10.1080/15548627.2016.1208889

32. Friedman SL, Neuschwander-Tetri BA, Rinella M, and Sanyal AJ. Mechanisms of NAFLD development and therapeutic strategies. Nat Med. 2018;24(7):908922. Http://dx.doi.org/10.1038/s41591-018-0104-9

33. Li L, Fu J, Liu D, Sun J, Hou Y, Chen C, et al. Hepatocyte-specific Nrf2 deficiency mitigates high-fat diet-induced hepatic steatosis: Involvement of reduced PPARy expression. Redox Biol. 2020;30:101412. Http://dx.doi.org/10.1016/j.redox.2019.101412

34. Meakin PJ, Chowdhry S, Sharma RS, Ashford FB, Walsh SV, McCrimmon RJ, et al. Susceptibility of Nrf2-null mice to steatohepatitis and cirrhosis upon consumption of a high-fat diet is associated with oxidative stress, perturbation of the unfolded protein response, and disturbance in the expression of metabolic enzymes but not with insulin resistance. Mol Cell Biol. 2014;34(17):33053320. Http://dx.doi.org/10.1128/mcb.00677-14

35. Liu Z, Dou W, Ni Z, Wen Q, Zhang R, Qin M, et al. Deletion of Nrf2 leads to hepatic insulin resistance via the activation of NF-KB in mice fed a high-fat diet. Mol Med Rep. 2016;14(2):1323- 
36. Shin S, Wakabayashi J, Yates MS, Wakabayashi N, Dolan PM, Aja S, et al. Role of Nrf2 in prevention of high-fat diet-induced obesity by synthetic triterpenoid CDDO-imidazolide. Eur J Pharmacol. 2009;620(1-3):138-144. Http://dx.doi.org/10.1016/j.ejphar.2009.08.022

37. Chartoumpekis DV, Palliyaguru DL, Wakabayashi N, Fazzari M, Khoo NKH, Schopfer FJ, et al. Nrf2 deletion from adipocytes, but not hepatocytes, potentiates systemic metabolic dysfunction after longterm high-fat diet-induced obesity in mice. Am J Physiol Endocrinol Metab. 2018;315(2):E180e195. Http://dx.doi.org/10.1152/ajpendo.00311.2017

38. Kim YR, Lee EJ, Shin KO, Kim MH, Pewzner-Jung Y, Lee YM, et al. Hepatic triglyceride accumulation via endoplasmic reticulum stress-induced SREBP-1 activation is regulated by ceramide synthases. Exp Mol Med. 2019;51(11):1-16. Http://dx.doi.org/10.1038/s12276-019-0340-1

39. Sun X, Li X, Jia H, Wang H, Shui G, Qin Y, et al. Nuclear Factor E2-Related Factor 2 Mediates Oxidative Stress-Induced Lipid Accumulation in Adipocytes by Increasing Adipogenesis and Decreasing Lipolysis. Antioxid Redox Signal. 2020;32(3):173-192. Http://dx.doi.org/10.1089/ars.2019.7769

40. Tanaka S, Hikita H, Tatsumi T, Sakamori R, Nozaki Y, Sakane S, et al. Rubicon inhibits autophagy and accelerates hepatocyte apoptosis and lipid accumulation in nonalcoholic fatty liver disease in mice. Hepatology. 2016;64(6):1994-2014. Http://dx.doi.org/10.1002/hep.28820

41. Kim YS, Nam HJ, Han CY, Joo MS, Jang K, Jun DW, et al. Liver X Receptor Alpha Activation Inhibits Autophagy and Lipophagy in Hepatocytes by Dysregulating Autophagy-Related 4B Cysteine Peptidase and Rab-8B, Reducing Mitochondrial Fuel Oxidation. Hepatology. 2021;73(4):1307-

1326. Http://dx.doi.org/10.1002/hep.31423

42. Singh R, Kaushik S, Wang Y, Xiang Y, Novak I, Komatsu M, et al. Autophagy regulates lipid metabolism. Nature. 2009;458(7242):1131-1135. Http://dx.doi.org/10.1038/nature07976

43. Arab JP, Arrese M, and Trauner M. Recent Insights into the Pathogenesis of Nonalcoholic Fatty Liver Disease. Annu Rev Pathol. 2018;13:321-350. Http://dx.doi.org/10.1146/annurev-pathol-020117043617

44. Wang J, Liu Z, Hu T, Han L, Yu S, Yao Y, et al. Nrf2 promotes progression of non-small cell lung cancer through activating autophagy. Cell Cycle. 2017;16(11):1053-

1062. Http://dx.doi.org/10.1080/15384101.2017.1312224

45. Wang Y, Zhang J, Huang ZH, Huang XH, Zheng WB, Yin XF, et al. Isodeoxyelephantopin induces protective autophagy in lung cancer cells via Nrf2-p62-keap1 feedback loop. Cell Death Dis. 2017;8(6):e2876. Http://dx.doi.org/10.1038/cddis.2017.265 
46. Huang DD, Yan XL, Fan SD, Chen XY, Yan JY, Dong QT, et al. Nrf2 deficiency promotes the increasing trend of autophagy during aging in skeletal muscle: a potential mechanism for the development of sarcopenia. Aging (Albany NY). 2020;12(7):59775991. Http://dx.doi.org/10.18632/aging. 102990

47. Tang M, Ji C, Pallo S, Rahman I, and Johnson GVW. Nrf2 mediates the expression of BAG3 and autophagy cargo adaptor proteins and tau clearance in an age-dependent manner. Neurobiol Aging. 2018;63:128-139. Http://dx.doi.org/10.1016/j.neurobiolaging.2017.12.001

48. Kitaoka Y, Takeda K, Tamura Y, Fujimaki S, Takemasa T, and Hatta H. Nrf2 deficiency does not affect denervation-induced alterations in mitochondrial fission and fusion proteins in skeletal muscle. Physiol Rep. 2016;4(24)Http://dx.doi.org/10.14814/phy2.13064

49. Saftig P, and Klumperman J. Lysosome biogenesis and lysosomal membrane proteins: trafficking meets function. Nat Rev Mol Cell Biol. 2009;10(9):623-635. Http://dx.doi.org/10.1038/nrm2745

50. Tang JX, Li J, Cheng JM, Hu B, Sun TC, Li XY, et al. Requirement for CCNB1 in mouse spermatogenesis. Cell Death Dis. 2017;8(10):e3142. Http://dx.doi.org/10.1038/cddis.2017.555

\section{Figures}


A

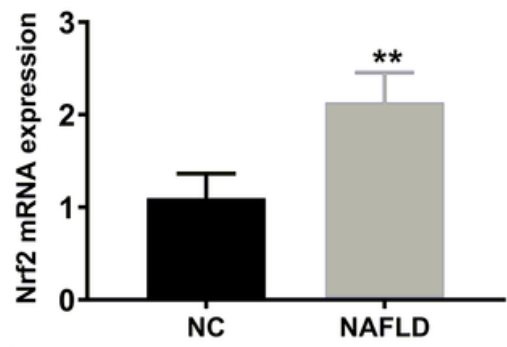

C

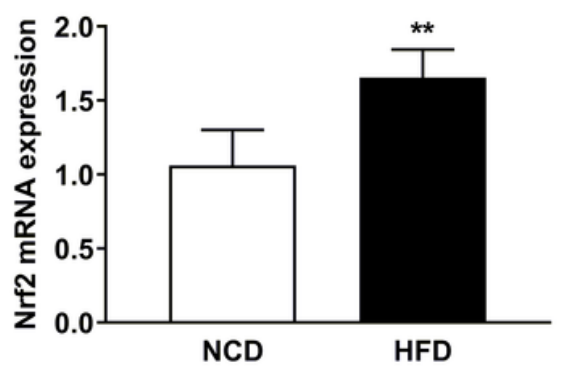

E

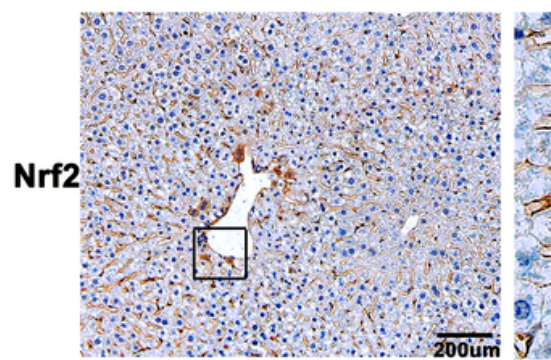

F

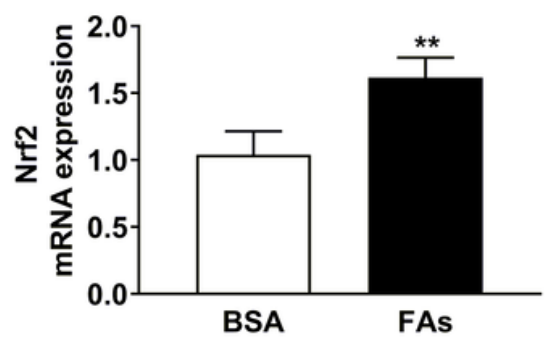

B

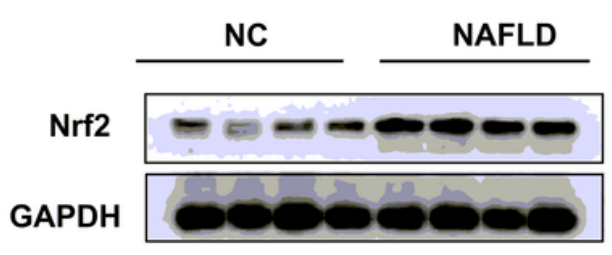

D

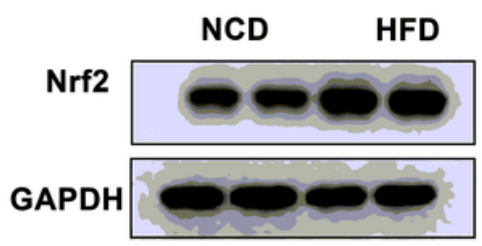

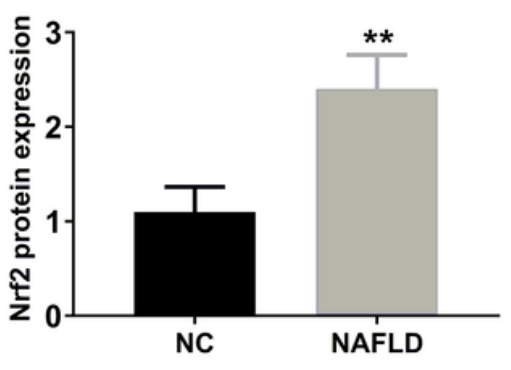

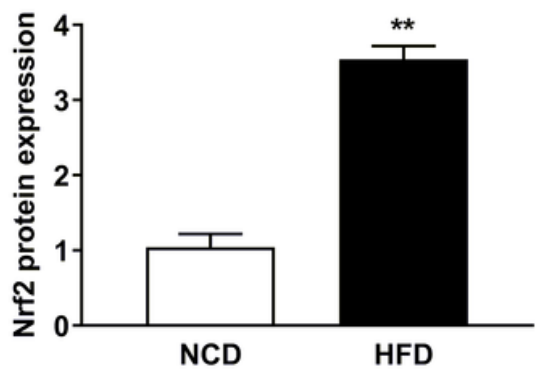

HFD

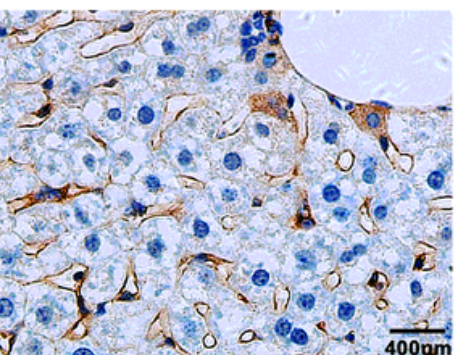

G
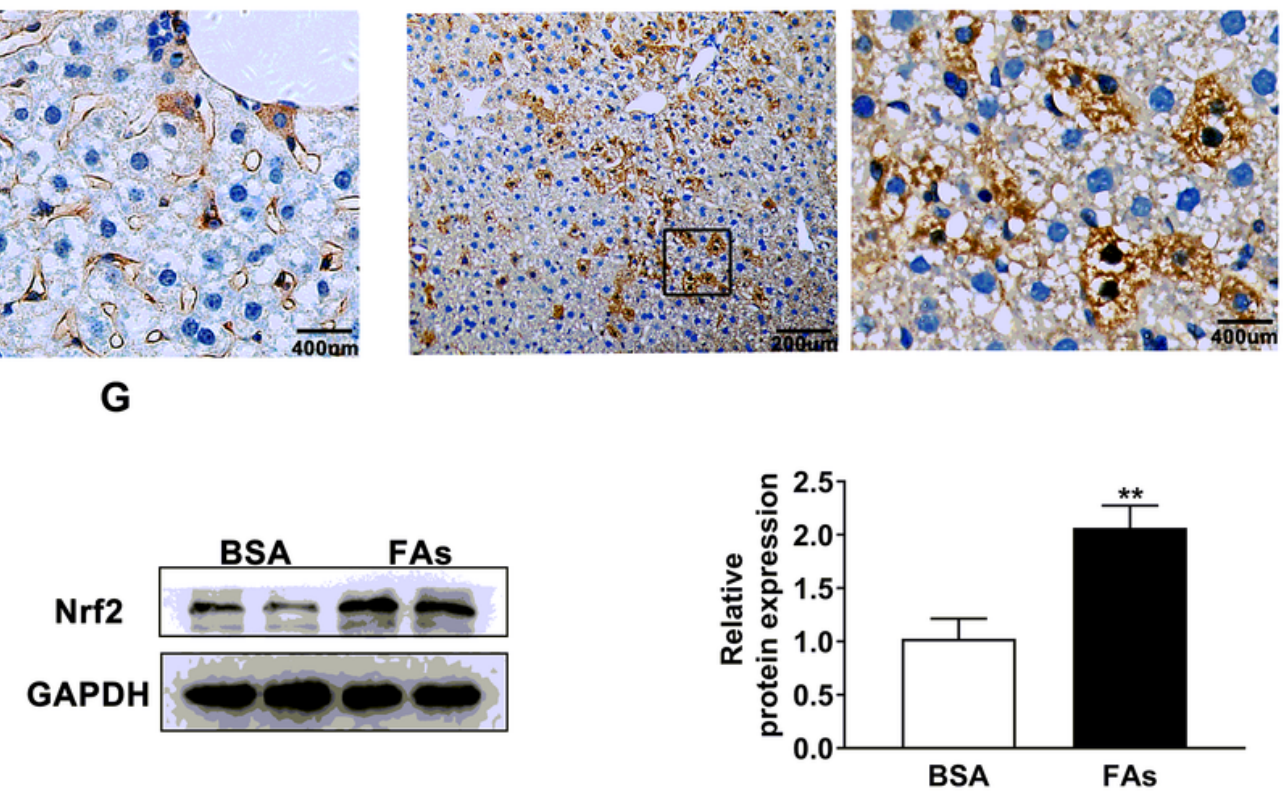

Figure 1

Hepatic Nrf2 is up-regulated in patients with NAFLD and obese mice. (A, B) Nrf2 mRNA (A) and protein (B) expression in the liver of patients with NAFLD. (c, d) Nrf2 mRNA (C) and protein (D) expression in the liver of NCD- or HFD-fed WT mice. (E) IHC staining of liver sections from NCD- or HFD-fed C57BL/6J mice. (F, G) MPHs from WT mice were treated with BSA or FAs for 24h. Nrf2 mRNA (F) and protein (G) expression. NC, normal controls; NCD, normal chow diet; HFD, high-fat diet; NAFLD, non-alcoholic fatty liver disease; 
MPHs, mouse primary hepatocytes. The data are expressed as means \pm SEM. western blot: $n=3-4 /$ group; animal experiments: $n=5-6 /$ group. ${ }^{*} p<0.01$ vs. NC, NCD, or BSA.

A

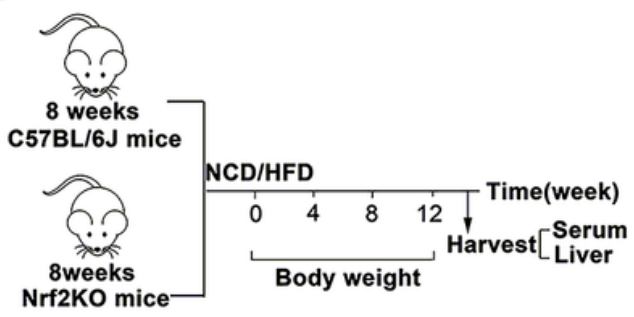

D

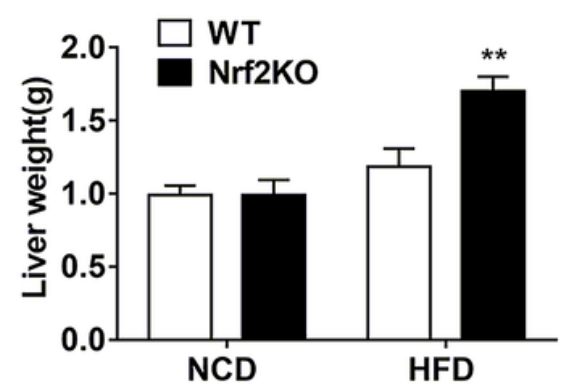

G

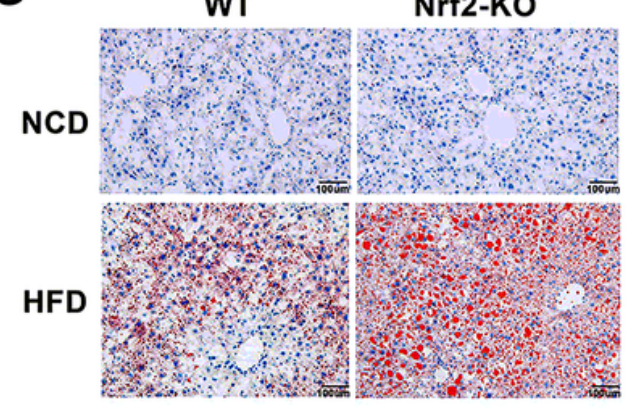

J

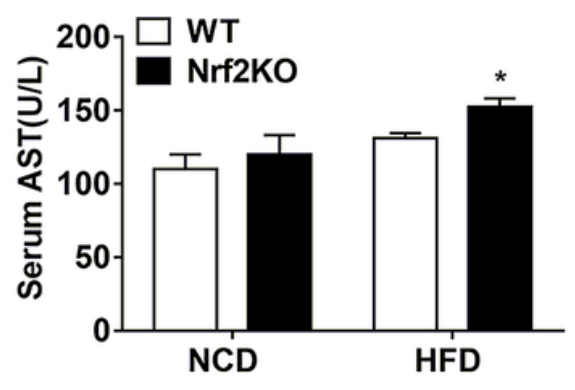

B

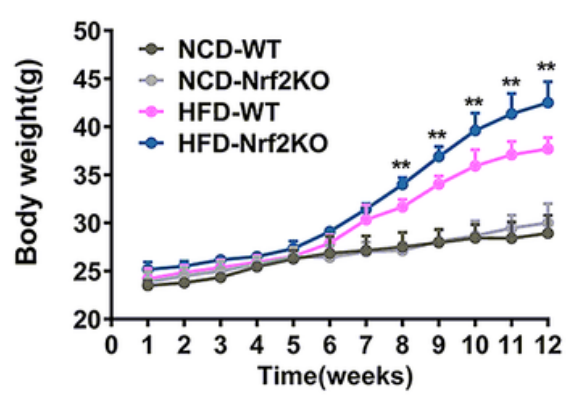

E

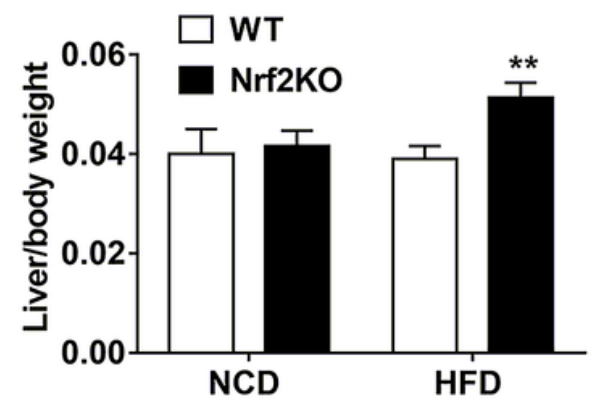

H

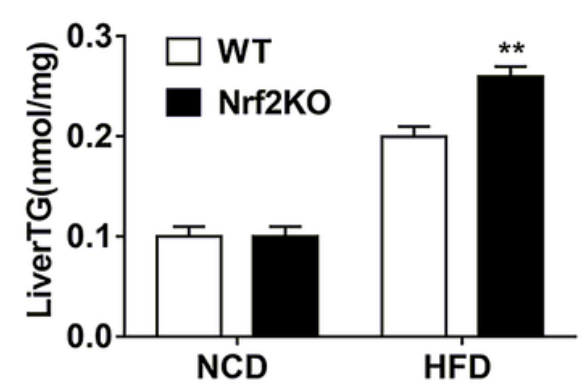

K

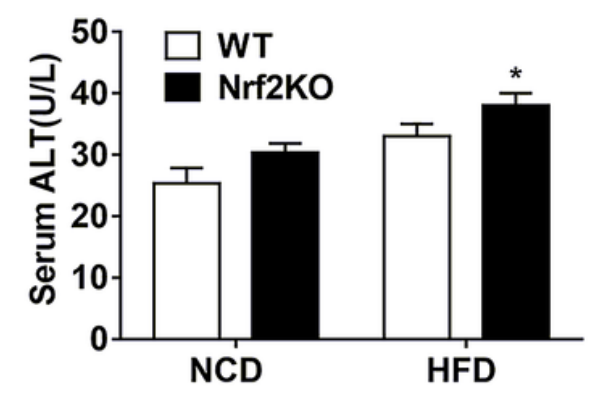

C

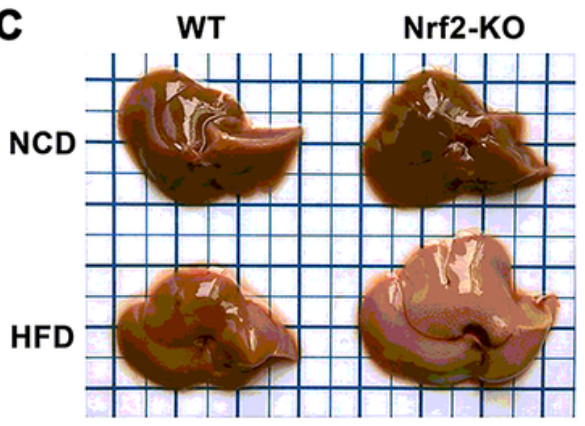

F

NCD

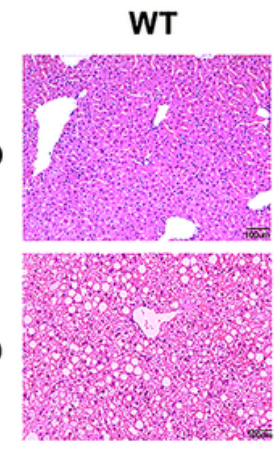

Nrf2-KO

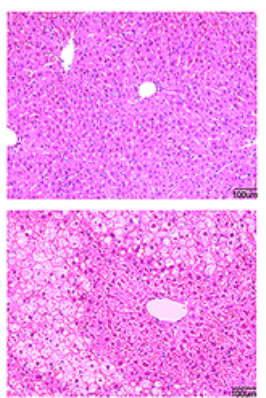

I

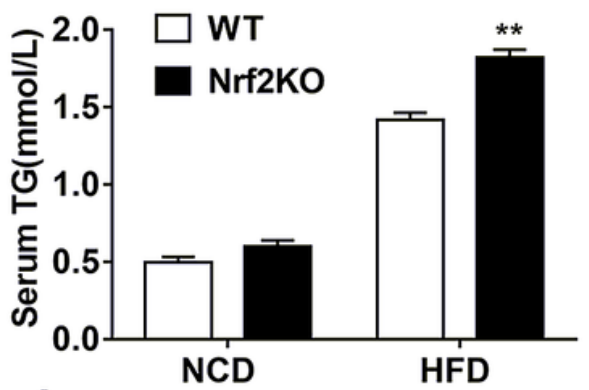

L

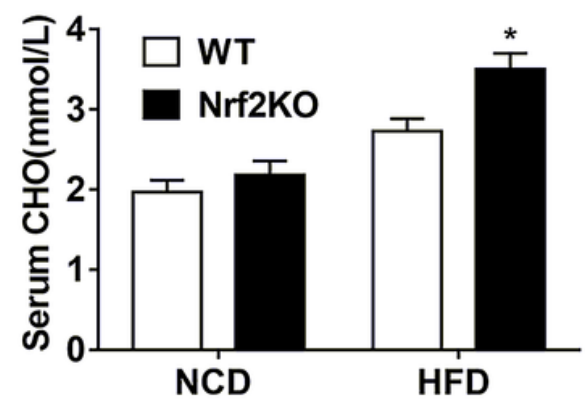

Figure 2

Nrf2 deficiency facilitates hepatic steatosis induced by HFD. 8-week-old WT and Nrf2 KO mice were fed an HFD or NCD for 12 weeks. (A) Schematic diagram of experiment design. (B) Body weight. (C) Representative livers at the macroscopic examination. (D) Liver weight. (E) Liver weight/body weight. (FG) H\&E(f) and Oil Red O (g) staining of liver sections. (H) Hepatic TG contents. (I) Serum TG levels. (J) Serum AST levels. (K) Serum ALT levels. (L) Serum CHO levels. HFD, high-fat diet; NCD, normal chow diet; 
TG, triglyceride; AST, aspartate transaminase; ALT, serum alanine transaminase; $\mathrm{CHO}$, serum cholesterol. Data are expressed as means \pm SEM. western blot: $n=3$ /group; animal experiments: $n=5$-6/group. * $p<$ $0.05, * * p<0.01$ vs. HFD-fed WT.

A

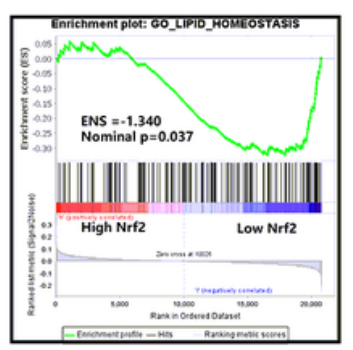

B

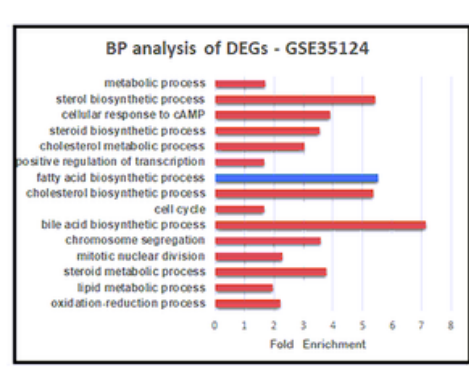

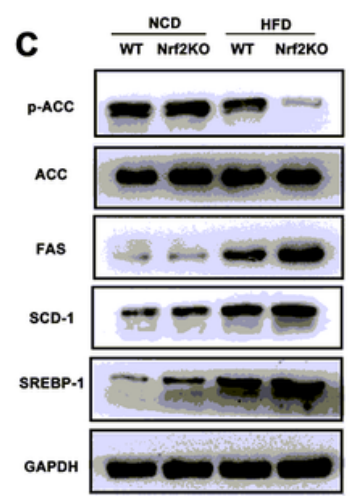

$\mathbf{F}$

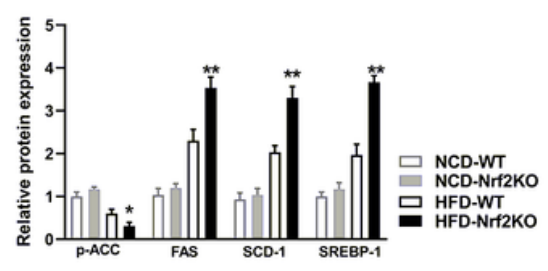

G
D

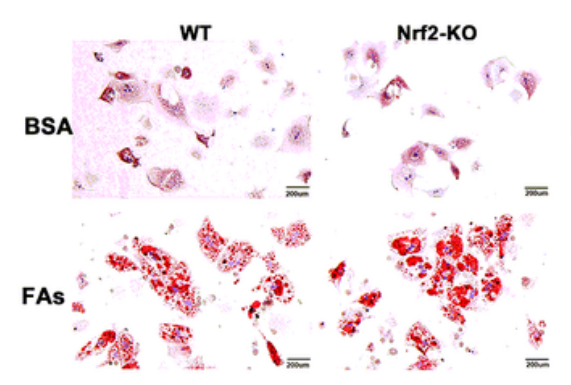

E

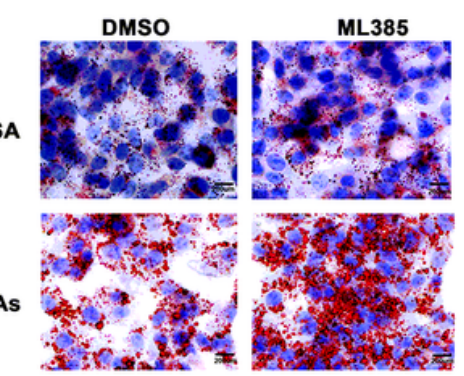

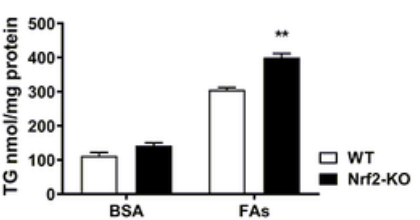

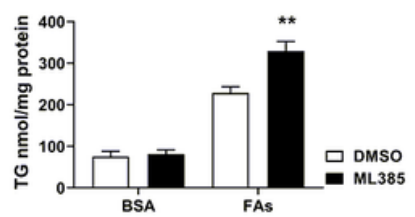

H

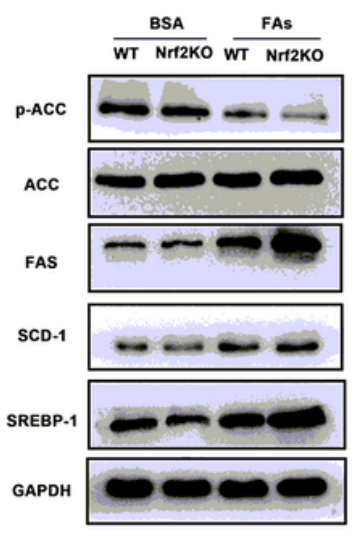

I

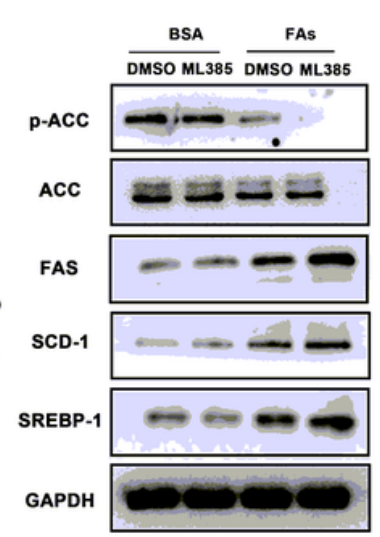

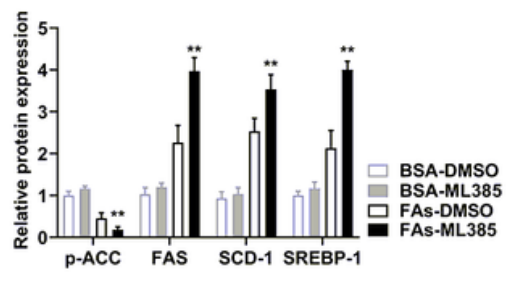

\section{Figure 3}

Effects of Nrf2 deficiency on lipogenesis in the livers. (A) GSEA enrichment analysis indicating a relationship between Nrf2 expression and lipid homeostasis. (B) BP enrichment analysis from the GSE35124 dataset. (C) 8-week-old WT and Nrf2 KO mice were fed an HFD or NCD for 12 weeks. Immunoblot analysis of gene expression in the liver. (D-L) MPHs from WT and Nrf2 KO mice and HepG2 cells were incubated with BSA or FAs for $24 \mathrm{~h}$. Oil red $O$ staining (D, E), TG contents (F, G), and Immunoblot analysis of gene expression $(\mathrm{H}, \mathrm{I})$. MPH, mouse primary hepatocyte; TG, triglyceride; FAs, fatty acid mixture; Data are expressed as means \pm SEM. western blot: $n=3-4$ /group; animal experiments: $n=5$ 6/group. * $p<0.05$, ** $p<0.01$ vs. HFD-WT, FAs-WT or FAs-DMSO. 
A

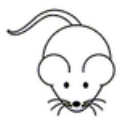

8 weeks

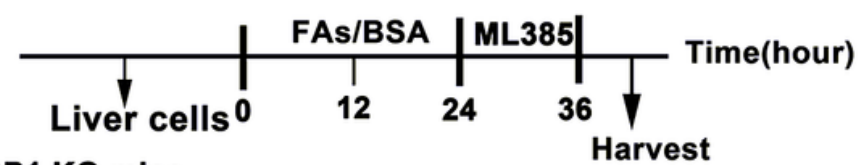

C57BL/6J or SREBP1 KO mice
B

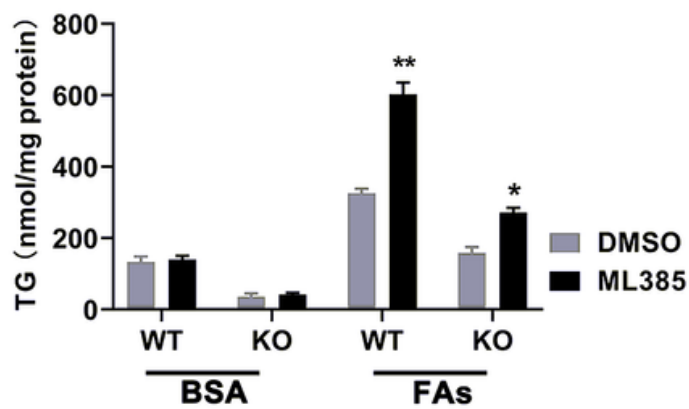

FAs

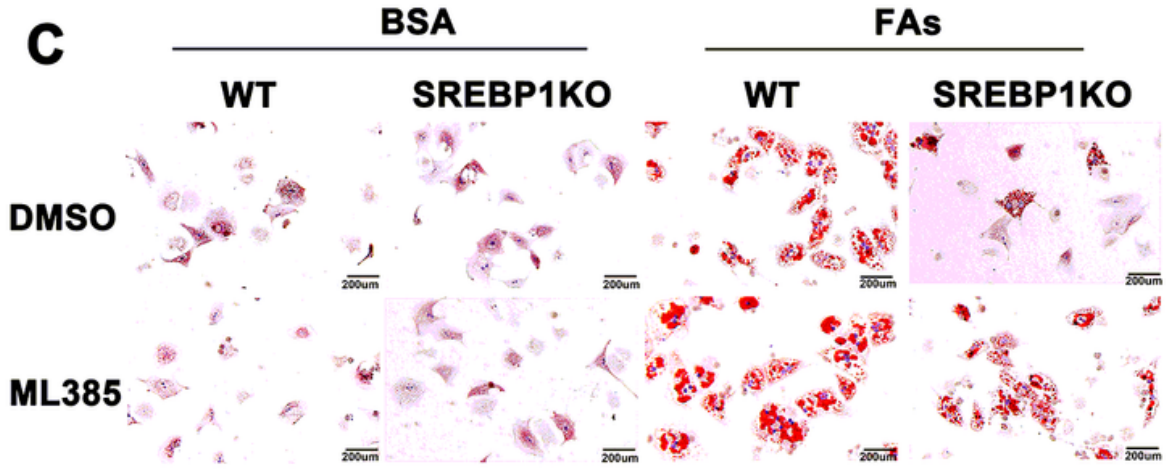

D

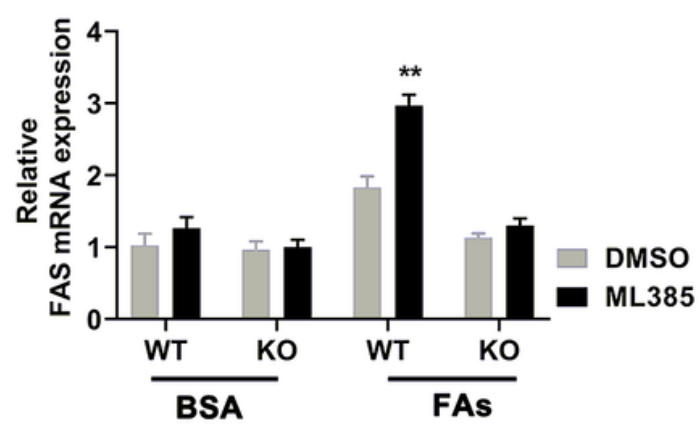

$\mathbf{E}$

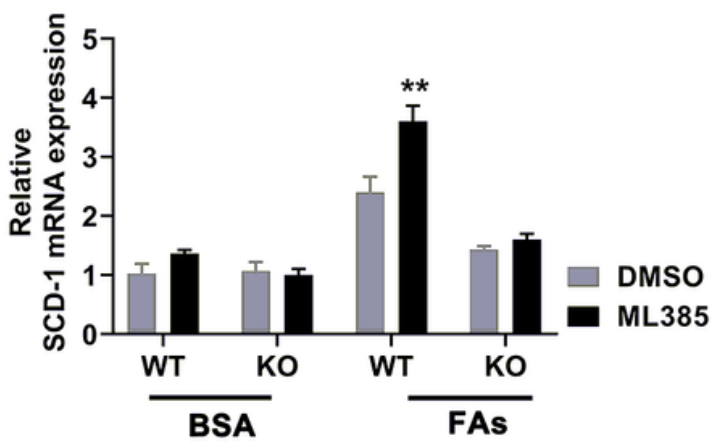

Figure 4

SREBP-1c is needed for the effect of Nrf2 on lipogenesis. (A) MPHs from WT and SREBP-1c KO mice were incubated with BSA or FAs for 24h and then treated with DMSO or ML385. (B) TG content. (C) Oil red 0 staining. (D) FAS and (E) SCD-1 mRNA expression. ML385, an Nrf2 inhibitor; Data are expressed as means \pm SEM from three independent experiments. ${ }^{*} p<0.05$, ${ }^{*} p<0.01$ vs. FAs- DMSO. 
A
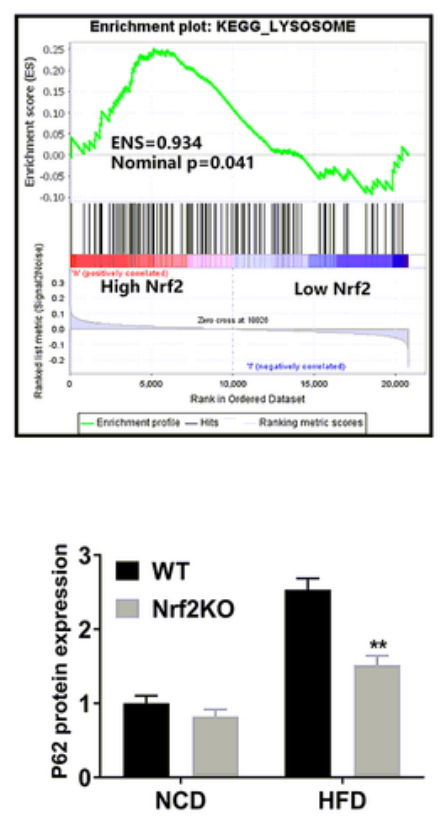

B

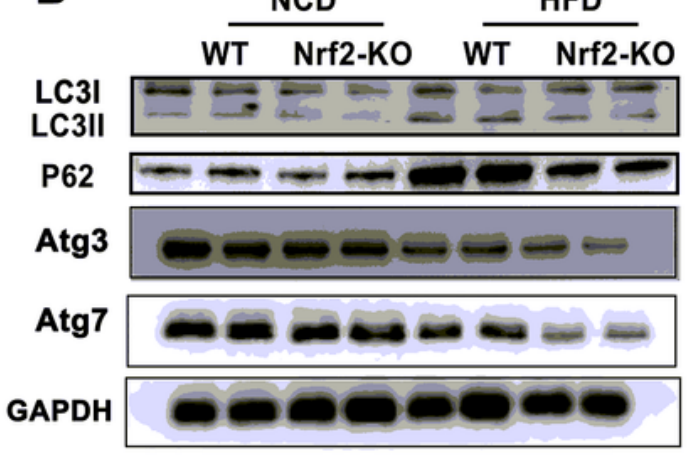

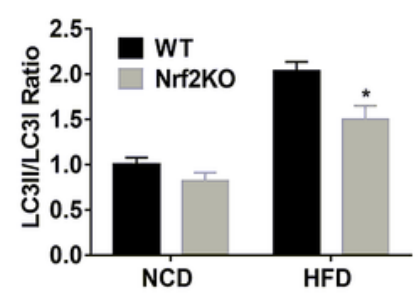
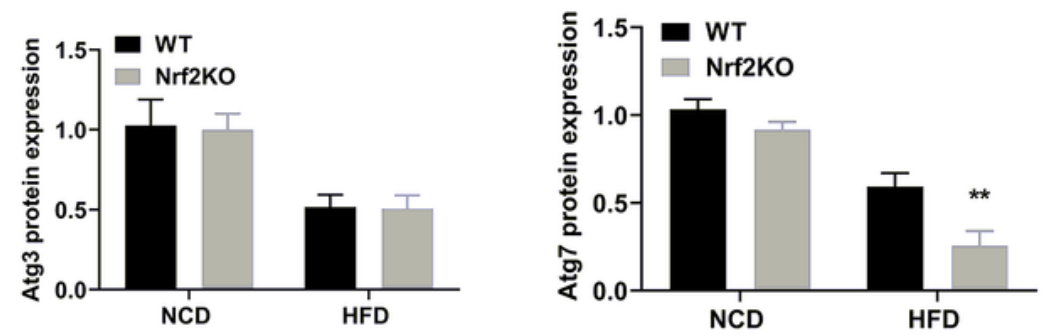

C
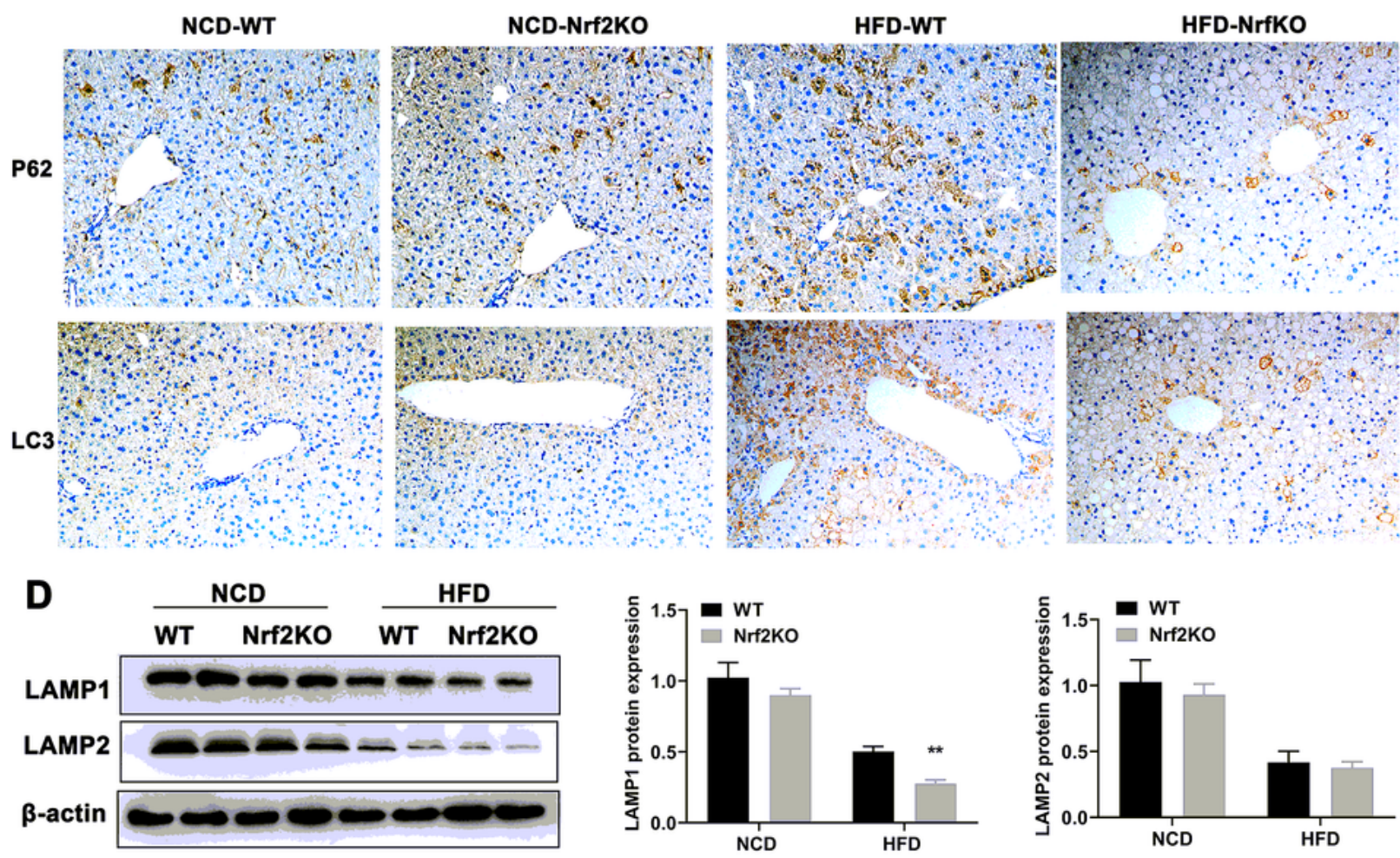

Figure 5

Nrf2 deficiency attenuates autophagy in the liver of mice fed an HFD. (A) GSEA enrichment analysis indicating the relationship between Nrf2 expression and lysosome function. (B-D) Male Nrf2 KO and WT mice were fed an HFD or NCD for 12 weeks. (B) LC3II, p62 and Atg protein expression in the livers. (C) IHC staining of liver sections for LC3 and p62. (D) LAMP1 and LAMP2 protein expression in the liver. Data are 
expressed as means \pm SEM. western blot: $n=3$ /group; animal experiments: $n=5-6 /$ group. * $p<0.01$, ** $p$ $<0.01$ vs. HFD-WT.
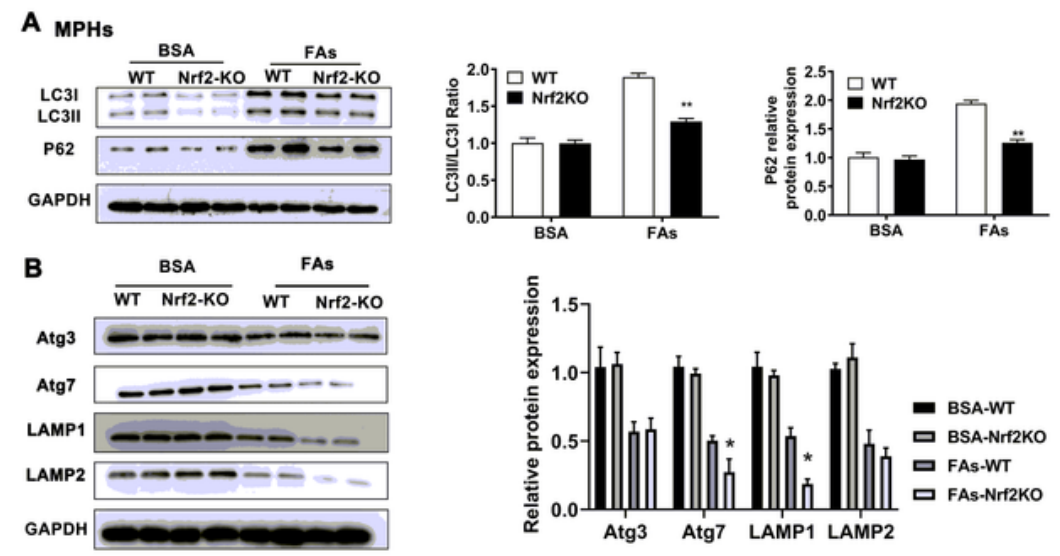

\section{HepG2}

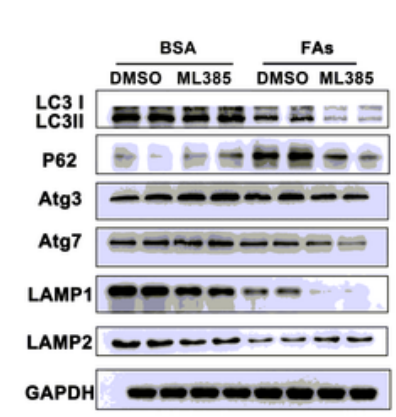

D MPHs
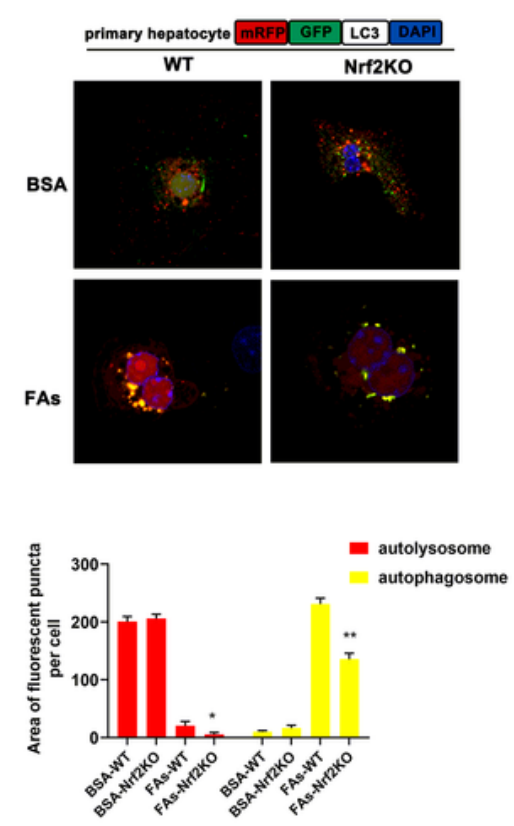
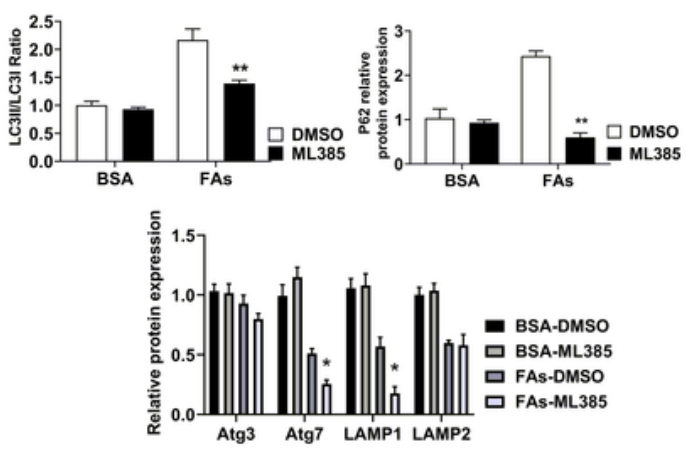

HepG2
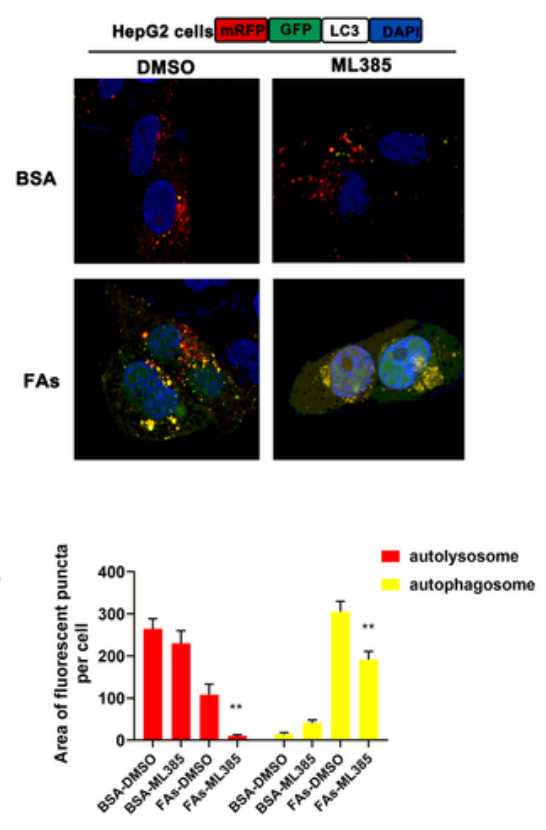

Figure 6

Nrf2 deficiency attenuates autophagy in vitro. (A, B) MPHs from Nrf2 KO and WT mice were treated with FAs or BSA for $24 \mathrm{~h}$. (A) p62 protein expression and LC3II densitometry. (B) Atg and LAMP expression at protein levels. (C) HepG2 cells were incubated with BSA or FAs for $24 \mathrm{~h}$ and then treated with DMSO or 
ML385. LC3II densitometry, p62, Atg and LAMP protein expression; (D) HepG2 cells and MPHs were transfected with Ad-mRFP-GFP-LC3 and then exposed to BSA or FAs for 24h. Confocal images and quantification of LC3 reporter; All images were obtained using a 90× objective. MPH, mouse primary hepatocyte; FAs, fatty acid mixture. Data are expressed as means \pm SEM from three independent experiments. ${ }^{*} p<0.01,{ }^{* *} p<0.01$ vs. FAs-WT or FAs-DMSO.

A

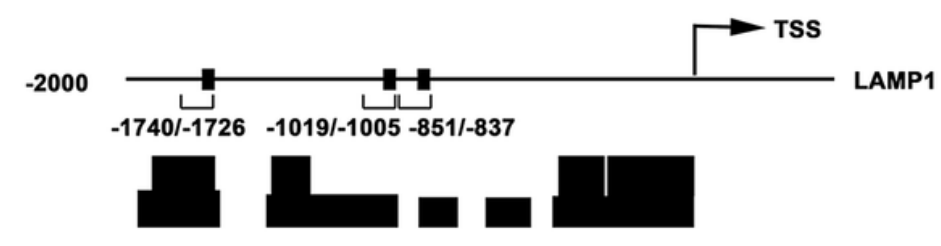

C
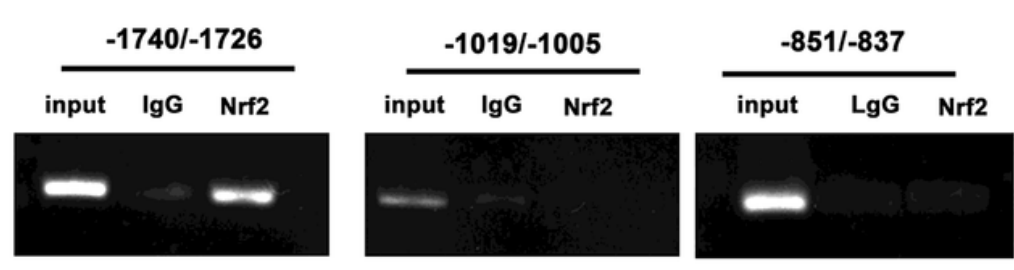

B
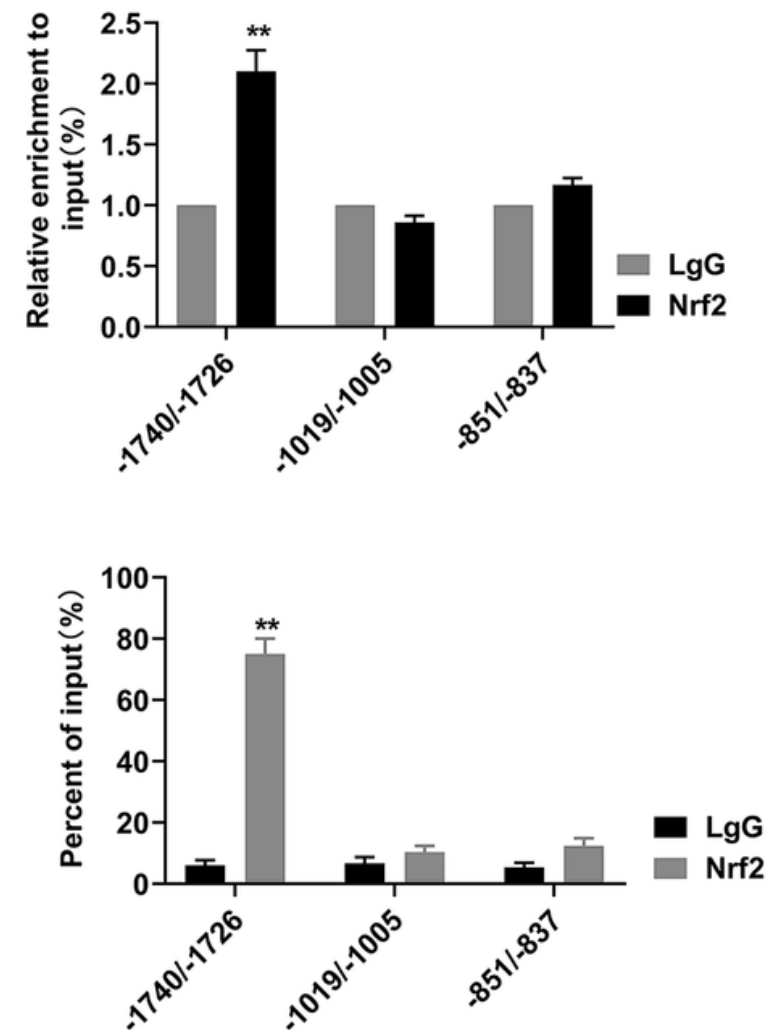

D

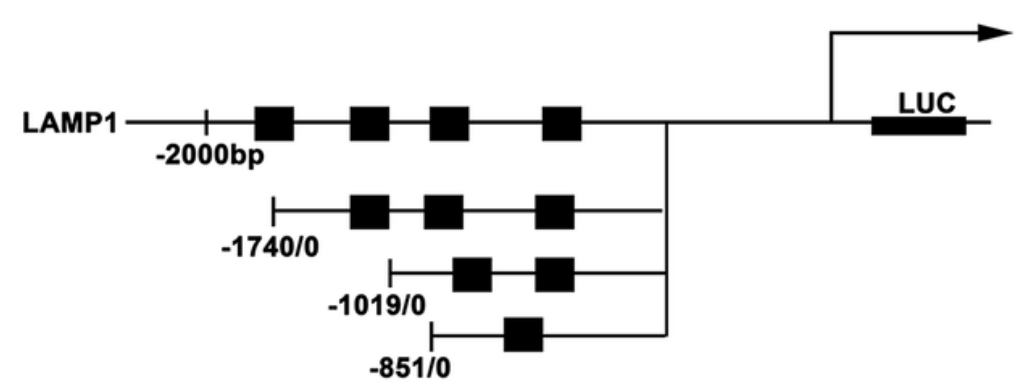

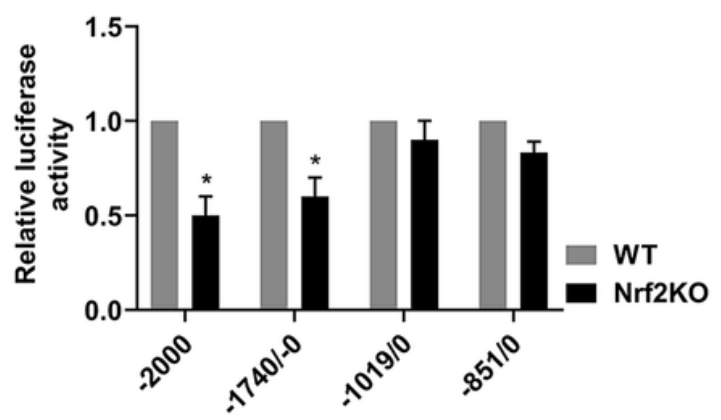

Figure 7

Nrf2 inhibits autophagy via the transcriptional regulation of LAMP1 promoter. (A) A schematic diagram for predicting the binding site of Nrf2 and LAMP1 promoter. (B) ChIP-qPCR analysis of the binding region of Nrf2 and the LAMP1 promoter. (C) Gel image and quantitative analysis of the ChIP DNA. (D) Luciferase reporter assay of LAMP1 transcriptional activity. Each bar represents the mean \pm SEM of three independent experiments. ${ }^{\star} \mathrm{p}<0.05,{ }^{*} \mathrm{p}<0.01 \mathrm{vs}$. IgG or WT. 


\section{Supplementary Files}

This is a list of supplementary files associated with this preprint. Click to download.

- originaldata.pdf

- OnlineSupFig1.png

- OnlineFigs2.png 